\title{
Sequential Decisions from Sampling: Inductive Generation of Stopping Decisions Using Instance-Based Learning Theory
}

\author{
Cleotilde Gonzalez and Palvi Aggarwal \\ Dynamic Decision Making Laboratory \\ Department of Social and Decision Sciences \\ Carnegie Mellon University, Pittsburgh, PA
}

Keywords: decisions from experience, sampling, instance based learning theory, satisficing. Prediction error.

Words for subject index: sampling paradigm, decisions from experience, instance based learning theory, IBL model, Prediction error. 


\begin{abstract}
Sequential decisions from sampling are common in daily life: we often explore alternatives sequentially, decide when to stop such exploration process, and use the experience acquired during sampling to make a choice for what is expected to be the best option. In decisions from experience, theories of sampling and experiential choice are unable to explain the decision of when to stop the sequential exploration of alternatives. In this chapter, we propose a mechanism to inductively generate stopping decisions, and we demonstrate its plausibility in a large and diverse human data set of the binary choice sampling paradigm. Our proposed stopping mechanism relies on the choice process of a theory of experiential choice, InstanceBased Learning Theory (IBLT). The new stopping mechanism tracks the relative prediction errors of the two options during sampling, and stops when such difference is close to zero. Our results from simulation are able to accurately predict human stopping decisions distributions in the dataset. This model provides an integrated theoretical account of decisions from experience, where the stopping decisions are generated inductively from the sampling process.
\end{abstract}

\title{
Introduction
}

Decisions that we make in many naturalistic situations involve a sequential process of sampling the available options before making a real choice (Hertwig et al., 2004; Gonzalez \& Dutt, 2016). For example, in selecting candidates for a job, we might evaluate the applicants sequentially, keeping in mind those most promising candidates, and deciding to stop the evaluation of candidates once we have enough evidence that our top candidate should be hired. Another example may involve the selection of a house for rent. The options available in the market may be visited sequentially, and we decide when to stop considering new options when we have a house that is good enough. Another example may be that of selecting a suit to 
purchase for a special event; multiple suits may be tried on in the fitting room, and we decide when to stop trying out suits when we have at least one that is appropriate for the event. In all these situations we evaluate each of the alternatives sequentially, decide when to stop the sampling process based on the information accumulated, and use the experience acquired during sampling to make a definite choice for what we think is the current best option.

The type of situation in which we explore potential alternatives before we make a choice has been studied in an abstract paradigm of experience-based choice between risky monetary gambles, referred to as the "Sampling Paradigm" in Decisions from Experience (DfE) research (Hertwig \& Erev, 2009; Hertwig et al., 2004). In the sampling paradigm, individuals decide how many outcomes to observe from a set of options of uncertain value before making a choice. Participants make one selection among the options available each time, and they observe the outcome which is drawn from an underlying payoff distribution in the option selected. When the person decides to stop the sampling process, a decision is made for one of the options, based on their experienced values during sampling.

A robust phenomenon that emerged from DfE research, with important theoretical implications, is the Description-Experience Gap (DE Gap). The DE Gap suggests that decisions made on the basis of experience contrast directly with decisions made based on pure description of the alternatives (Hertwig et al., 2004; Gonzalez \& Dutt, 2011; Wulff, et al., 2018). A common explanation of the DE Gap is a "sampling error": when people rely on experience they tend to rely on a small number of samples (i.e., sample size), which will result in observed distributions of outcomes that are distorted and not reflective of the true distributions (Hertwig \& Pleskac, 2010; Hertwig et al., 2004; Hau, Pleskac, Kiefer \& Hertwig, 2008). A major question in DfE 
research is therefore, how do people decide the number of samples to observe (i.e., when to stop sampling?).

A large amount of research has investigated choice in the sampling paradigm. For example, to address the question of how do people decide their sampling size, researchers have looked at moderators of the sampling size including: the structure of the problems and the variability of the options (e.g., a choice between two risky alternatives or between a risky and a safe option) (Wulff, et al., 2018; Gonzalez \& Mehlhorn, 2016), the time available and motivations to sample more or less (Hau et al. 2008), and the domain of the problem (e.g., whether gains, losses or a mix of outcomes are involved) (Lejarraga, Hertwig \& Gonzalez, 2012; Gonzalez \& Mehlhorn, 2016). However, as concluded in a recent meta-analysis by Wulff et al., (2018), theories of sampling and experiential choice are not capable of explaining the sequential sampling process and the final choice, while at the same time also explaining the decision of when to stop sampling. Our goal in this chapter is to offer a theoretical foundation to integrate the decision of the sampling size into a well-known general theory of the cognitive processes in experiential choice, Instance-Based Learning Theory (IBLT, Gonzalez, Lerch, \& Lebiere, 2003; Gonzalez \& Dutt, 2011).

\section{Models of DfE in the Sampling Paradigm}

In early DfE research of the sampling paradigm (Hertwig et al., 2004), researchers aimed at discovering the effect of experience acquired during sampling on the subsequent consequential choice. Thus, most models of DfE in the sampling paradigm focused on capturing the decision after sampling but not the choices during the sampling process itself. Furthermore, existent models of sampling vary greatly in the assumptions they make about the sampling process. A modeling competition held in 2009 by Erev and colleagues (Erev et al., 2010) illustrates these 
issues. The competition was designed to compare and select the best models of DfE (sampling paradigm and other paradigms as well). Contestant teams submitted computational models after fitting them to an "estimation" data set provided by the organizers. The models were compared based on how well they predicted a "competition" data set to which modelers did not have access to. The models submitted to the sampling paradigm illustrate the kind of broad and disparate assumptions these models make about the sampling process, including some models that assume a random selection of options during sampling (Erev et al., 2010). For example, the baseline model provided by the organizers, assumes a random sample of $k$ draws (a parameter fit to data) from each of the available options and the accumulation of outcomes based on the random sample of each option (Erev et al., 2010). None of the models submitted to the modeling competition present an integrated view of how the selection process is done during sampling and final choice, or the prediction of the sampling size distribution.

After the competition, Gonzalez and Dutt (2011) analyzed the models and presented an alternative model comparison, providing a summary of the highest ranking models that address the sampling paradigm in the Erev et al. (2010) competition. None of those models is able to predict the sampling process and sampling size decisions together. Gonzalez and Dutt (2011) provide a general model of DfE based on IBLT; a model that is be able to capture the process of human experiential choice, not only in the sampling paradigm but in other paradigms as well (Hertwig \& Erev, 2009). Using a quantitative model comparison, Gonzalez and Dutt (2011) demonstrated that the IBL model generalized well across experimental paradigms. Importantly, the IBL model was the only model able to predict the sequence of sampling selections that humans make during sampling as well as the final consequential choice after sampling. This was achieved using the same common cognitive mechanisms of IBLT, an achievement unique to this 
model. The generality of the IBL model of choice was further demonstrated in static and dynamic tasks (Lejarraga, Dutt \& Gonzalez, 2012), in tasks involving experience and descriptions (Lejarraga \& Gonzalez, 2011), in tasks involving teams (Lejarraga et al., 2014), and in two-choice social dilemmas (Gonzalez et al., 2015). This model is now considered as the most versatile and comprehensive model of DfE (Hertwig, 2015).

Despite the successful demonstrations of the generality of the IBL model, there is one question left unanswered in Gonzalez and Dutt (2011): how is the stopping decision made?. Gonzalez and Dutt (2011) used a binomial distribution of sample sizes to determine the sample size in each simulation ran with the IBL model. This distribution has shown to accurately represent the sample size distribution in humans (e.g., Wulff et al., 2018). In other words, the IBL model itself did not make a stopping decision, but it made the choices of which option during the sequential sampling process and the final choice based on the IBL mechanisms.

The particular question of how the decision of the sample size is made during the sampling process is largely unanswered. In well-known accumulation models, the sampling length depends on the relative evidence strength of the choice options (e.g., Busemeyer and Townsend, 1993). These models however, often rely on perceptual decision tasks and duration is measured in continuous time. In the sampling paradigm of DfE, the sampling size is discrete and it is unclear how the continuous duration would translate to the discrete number of samples. In the literature, there are a couple of papers that have aimed at proposing mechanisms for making stopping decisions in the sampling paradigm of DfE. First, Markant, et al., (2015) present a sequential sampling model that tries to explain the individual variations in the sample size. The model relies on Cumulative Prospect Theory (Tversky \& Khaneman, 1992) to determine the value of options. But it also adds a number of assumptions regarding transition probabilities 
during sampling, and the trajectory that a preference or relative value of the two options in a binary choice task (i.e., drift rate) (Markant et al., 2015). The number of samples drawn depend on the drift rate and on a threshold level of preference. Sampling stops when the threshold is reached. The model reported high accuracy in the choice proportions, and the distributions of sample size appear to be similar to those of human data. However, there was no quantification of the accuracy of the model in accounting for the sample size, together with the choices during and after sampling. Generally, the model is quite complicated and likely not cognitively plausible.

Second, Srivastava, Muller-Trede, Schrater, and Vul (2016) followed on the work of Markant et al. (2015) with a simpler model that relies on the difference of the expected values of the two options and on "volatility," representing the trend of the prediction error. An interesting insight from this paper is that the prediction error should decrease over samples and help determine when to stop sampling. Volatility is a cognitively plausible concept suggesting that as long as this prediction error increases and changes, humans might infer that more samples are required, and that stopping makes sense once the volatility converges to a low value. Srivastava et al. (2016) tested individual volatility in a simple additive model explaining about 20-30\% of the variance in human sampling sizes. The best fit correlation of sampling durations predicted by this model and human data is $\mathrm{R}=0.03$. They improved this model but unfortunately introduced some unrealistic assumptions regarding what the observer in the task knows (e.g., average difference of the options' expected values and cumulative volatility across the sequence).

Inspired by these efforts, we propose a mechanism to inductively generate the stopping decision using the IBL model of choice. We test such mechanism by comparing data generated through IBL simulations to human data in the largest and most diverse data set of the sampling paradigm known today (Wulff et al., 2018). 


\section{IBLT and the IBL Model of Choice.}

In making decisions from experience, we evaluate available alternatives sequentially, by comparing each alternative to choices we have made in the past, and act according to the best outcomes we have experienced. This idea was proposed and formalized in IBLT, a theory of how humans make decisions from experience in dynamic environments (Gonzalez, Lerch, \& Lebiere, 2003).

In IBLT's process, alternatives are evaluated sequentially by sampling the environment, comparing each available alternative to past choices from experience. Based on the similarity of the alternative's attributes, alternatives are evaluated by aggregating experienced outcomes, to determine their expected value. Ultimately, an option with the best expected value is selected and executed. After each sampling selection is made, a stopping decision is considered regarding whether to continue to sample information or to stop and make a choice for the current best option. Such process in IBLT is similar to the "satisficing" strategy that has been contrasted to “optimizing” strategies in decision making research (Simon, 1957; March \& Simon, 1958). In IBLT, stopping decisions are often determined by the time remaining to make a choice, which is dictated by the dynamics of the environment (Gonzalez, et al., 2003). Here we propose a way to formalize such satisficing mechanism in an IBL model of choice in which the sampling process happens over discrete samples rather than continuous time.

The mathematical formalizations used in the IBL model of choice have been published in multiple places (e.g., Lejarraga et al., 2012, Gonzalez \& Dutt, 2011, Gonzalez, 2013). However, for completeness, we present these general mechanisms here before introducing the satisficing mechanism, which is the novel contribution in this chapter. 


\section{IBL model of Choice}

An "instance" in IBLT is a memory unit that represents the potential alternatives

evaluated. Instances are memory representations consisting of three elements: a situation (a set of attributes that give a context to the decision, or state $S$ ); a decision (the action taken corresponding to an alternative in state $S$, or action $A$ ); and a utility (expected utility or experienced outcome $x$ of the action taken in a state). An option $k=(S, A)$ is defined by taking action $A$ in state $S$. In the binary choice task, there are two options (i.e., $k=2$ ), the instances do not have a context (attributes) $(S)$; thus they only consist of the action $(A)$ and the outcome $(x)$. For example, in the binary choice between: Option A: a .8 chance to get $\$ 4$ and .2 chance to get $\$ 0$, and Option B: get $\$ 3$ for sure; the instances are: $(A, 4),(A, 0)$, and $(B, 3)$.

At time $t$, assume that there are $n_{k, t}$ different generated instances $k, x_{i, k, t}$ for $i=1, \ldots, n_{k, t}$, corresponding to selecting $k$ and achieving outcome $x_{i, k, t}$. Each instance $i$ in memory has an "activation" value, defined in the ACT-R cognitive architecture, and which represents how readily available that information is in memory (Anderson et al., 2004). The activation of an instance is determined by similarity to past situations, recency, frequency, and noise. In the IBL model of choice, we consider a simplified version of the activation equation which only captures how recently and frequently instances are activated:

$$
A c t_{i, k, t}=\ln \left(\sum_{t^{\prime} \in T_{i, k, t}}\left(t-t^{\prime}\right)^{-d}\right)+\sigma \ln \frac{1-\xi_{i, k, t}}{\xi_{i, k, t}}
$$

where $d$ and $\sigma$ are the decay and noise parameters, respectively, and $T_{i, k, t} \subset\{0, \ldots, \mathrm{t}-1\}$ is the set of the previous timestamps in which the instance $i$ was observed. The rightmost term represents the Gaussian noise for capturing individual variation in activation, and $\xi_{\mathrm{i}, \mathrm{k}, \mathrm{t}}$ is a 
random number drawn from a uniform distribution $\mathrm{U}(0,1)$ at each time step and for each instance and option.

Activation of an instance $i$ is used to determine the probability of retrieval of an instance from memory. The probability of an instance $i$ is a function of its activation $A c t_{i, k, t}$ relative to the activation of all instances:

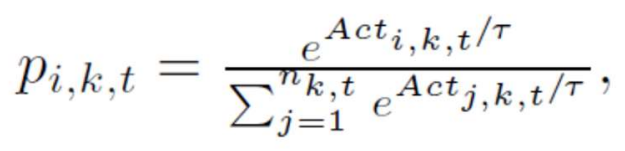

where $\tau$ is the Boltzmann constant (i.e., the "temperature") in the Boltzmann distribution. For simplicity, $\tau$ is often defined as a function of the same $\sigma$ used in the activation equation $\tau=\sigma \sqrt{2}$

The expected utility of option $k$ is calculated based on a mechanism called Blending, using the past experienced outcomes stored in each instance. In the IBL model of choice employs the Blending calculation as defined for choice tasks (Lejarraga et al., 2012; Gonzalez \& Dutt, 2011) $)^{1}$

$$
V_{k, t}=\sum_{i=1}^{n_{k, t}} p_{i, k, t} x_{i, k, t}
$$

Essentially, the Blending operation is the sum of all past experienced outcomes weighted by their probability of retrieval. Where $x_{i k t}$ is the outcome stored in an instance $i$ associated with choosing $k$; at time $t$. $p_{i k t}$ is the probability of retrieving the instance $\mathrm{i}$ from memory (Eq. 2$) . n_{k, t}$ is

\footnotetext{
${ }^{1}$ A more generalized formulation of the Blending equation can be found in Lebiere (1999).
} 
the number of instances stored in memory for option $k$ up to the last trial. The choice rule is to select the option that corresponds to the maximum blended value.

To begin the task, instances are initialized with an outcome value that is higher than those experienced in the task, which represents expectations and helps in initiating exploration of the options (Lejarraga et al., 2012). In the simulations presented in this chapter we do not fit the model's parameters in any way. We use parameters that are considered "default" in the ACT-R community: where $d=0.5$ and $\sigma=0.25$. Thus, the model data reported here, is generated from the IBL model simulations, which are pure predictions from the IBL model.

\section{A Satisficing Mechanism in the IBL Model of Choice.}

Similar to the idea of Srivastava et al., (2016), we propose that humans are able to determine the error in their predictions by observing the outcome from the option selected through immediate feedback. This error is then used to track the relative prediction errors of the two options during sampling. Intuitively, it is expected that the relative value of the prediction errors will decrease with learning, as the estimations through Blending converge. But clearly, the way such decline occurs will depend largely on the structure and variability of the environment. This proposed process in the IBL model is formalized as follows:

First, for each option $k$, we calculate the prediction error: the difference between the expected value (Blended value) of the option, $V_{k t}$, and the actual outcome obtained $x_{k t}$ at time $t$, we call this value the "Gap"2:

$$
\operatorname{Gap}_{k t}=\left|V_{k t}-x_{k t}\right|
$$

\footnotetext{
${ }^{2}$ A similar “Gap" concept was used in Gonzalez, et al., 2015 for IBL models of the Prisoner's Dilemma,
} where the actions of each player were determined by a dynamic expectation of the actions of the other player. 
Second, we calculate the relative prediction errors for the two options, $k=1$ and $k=2$, form time $t-1$ to time $t$, we call this value the $\delta G a p_{t}$ and is defined as follows:

$$
\delta \operatorname{Gap}_{t}=|| \operatorname{Gap}_{1 t}-\operatorname{Gap}_{1(t-1)}|-| \operatorname{Gap}_{2 t}-\operatorname{Gap}_{2(t-1)}||
$$

The $\delta G a p_{t}$ is expected to decrease over time, and the prediction is that sampling will stop at trial $t$, when the marginal value $\delta G a p_{t}$ is close to zero. But, as it will become clear later, the Wulff al (2018) data set contains a very diverse set of problems with very different values and the decline of the $\delta G a p_{t}$ can vary largely from problem to problem. To account for the large variability of problems in the data set with a single model, we made the stopping point during sampling a probabilistic process. We defined an increasing concave probability function (from 0.5 to 1 ) that will determine whether to actually stop every time the $\delta G_{a p}$ reaches a threshold value close to zero (we defined such a threshold value of 0.10 ). This probability (starting at 0.5 ) increases by a negative $\operatorname{logarithm}$ function $(-\log \mathrm{p}$, where $\mathrm{p}$ is previous probability) each time $t$ in $\delta G a p_{t}$ reaches the threshold, such that the sampling stops (with probability 1) after the threshold has been reached for the $7^{\text {th }}$ time.

\section{Data Set and Simulation Methods}

Wulff et al. (2018) obtained data sets from original authors of papers containing one or more experiments with the sampling paradigm. The data set details are reported in Wulff et al., (2018, Appendix A) and it was used to conduct a meta-analysis. The dataset is composed of 80 different papers with a total of 4,400 participants and 45,239 decisions from experience (we did not use the data of decision from description). Out of the 80 papers, 55 of them had experiments with autonomous sampling, where a stopping decision was made (participants decided how long to sample the two options). These data sets are the only ones relevant for testing the proposed satisficing mechanism in the IBL model. 
Thus, the full dataset used in the current study consisted of 40,246 participants; 1,219 unique different problems; 1,906 paper-problem pairs; and a total of 1,065,918 decisions from experience including the sampling decisions and the final choice. The number of outcomes in each of the two options vary across problems between 1 and 5; the features of the problems also vary, such as whether the two options are risky, or one option is risky and the other one is safe; or whether the problems involve gains, losses and mixed types of outcomes.

We observed that some experiments had less than 10 participants, giving very little and noisy data for analyses. Thus, we removed 865 such paper-problem pairs. The resulting data set consists of 39,111 participants, 610 unique different problems, 1,041 paper-problem pairs, and a total of 842,242 decisions from experience including sampling and final choice. When analyzing the number of samples in this dataset we found that the number of samples vary from 1 to 750 . We visualized the data using boxplots and observed outliers. Using the Inter Quartile Range (IQR) rule, we detected those observations above $\mathrm{Q}_{1+-}-1.5 * \mathrm{IQR}$ or below $\mathrm{Q}_{1--1.5} * \mathrm{IQR}$ and removed those outliers from the data.

After the outlier removal, the final dataset used for analyses consists of 37,106 participants, 610 unique different problems, 1,041 paper-problem pairs, and a total of 842,242 decisions from experience including sampling and final choice. In this data set the sample sizes range from 1 and 71 samples.

\section{IBL model's simulation methods.}

We ran equal number of simulated IBL agents i.e., 39,111 on the 610 problems observed in the human data before removing the outliers. It is important to remember that we used the default decay $(d=0.5)$ and noise $(\sigma=0.25)$ parameters in the model, that is, we did not use the 
human data to inform the model and did not fit the parameters of the model to the human data. In other words, the simulation results are pure predictions from the IBL theory.

The IBL model makes a decision of which of the two options to sample in each trial, according to the Blended value (Equation 3). After each sample, the model makes the decision of whether to stop sampling based on the calculation of the marginal value of the two options $\left(\delta G a p_{t}\right)$ and threshold (Equation 5). When the model reaches the threshold for the first time, it has a 0.5 probability of stopping, and this probability increases every time the threshold is reached. If the model decides to stop sampling, it then makes the final choice after sampling, according to the Blended value of the two options accumulated during sampling (Equation 3).

The $\delta G_{a p}$ (Equation 5) lets the IBL agents to keep sampling after the threshold has reached up to 7 times (i.e., until the probability of stopping is 1), or when the maximum number of samples are reached. We used 750 as the maximum number of samples allowed in the model and a threshold of 0.10 , an arbitrary value close to zero. After running the simulations, we performed an outlier analysis on the simulation data, using the similar criteria as that used in human data. We used 71 samples as cutoff in IBL model, i.e., we removed those agent data whose sample size was greater than 71 . The IBL dataset resulting from this process has 33,509 agents, 610 unique different problems, 1,041 paper-problem pairs, and a total of 715,732 decisions from experience including sampling and final choice.

\section{Results}

We concentrate our analyses on the stopping decisions reflected in the sample size, for the 1,041 paper-problem pairs in the empirical data set and in the model dataset. The sample size is the total number of selections made by participants (or by agents in the model) during sampling and up to the stopping point, before making a choice. 


\section{Stopping Decisions: Sample size distributions.}

Figure 1 presents the distributions of sample sizes in human data compared to the IBL model. Panel A presents these sample size distributions across all the data. A geometric distribution represents the human sample-size distribution (Geometric $(p=.042)$ with $\chi^{2}(7)=$ $2877, p<.001)$. This geometric distribution is heavily right-tailed, the mean sample size is 22.7 $(\mathrm{SD}=16.01)$, and the median sample size is 20 samples. Similarly, a geometric distribution also represents the IBL model's sample-size distribution accurately (Geometric $(p=.045)$ with $\chi^{2}(7)$ $=9626, p<.001)$. The model's distribution's mean sample size is $21.36(\mathrm{SD}=13.62)$, and the median is 19 samples. The chi-square test signifies that human distribution and model sample distribution is strongly associated $\left(\chi^{2}(7)=3765, p<.001\right)$.

Panel B of Figure 1 shows the sample size distributions according to the type of risk in the options. These are Risky-Risky and Risky-Safe type problems. Human participants sample more in Risky-Risky problems (median $=21$, mean $=24.87, \mathrm{SD}=16.03)$ compared to RiskySafe problems $($ median $=13$, mean $=17.11, \mathrm{SD}=14.38)$. The IBL agents also sampled more in Risky-Risky problems $($ median $=21$, mean $=23.57, \mathrm{SD}=15.21)$ compared to Risky-Safe problems $($ median $=17$, mean $=16.71, \mathrm{SD}=8.91)$. The median sample size in the model is slightly higher than the human data in the Risky-Safe problems, but generally, the predictions of the model are very accurate. The chi-square tests signify that human sample distribution and model sample distribution are strongly associated in Risky-Safe problems $\left(\chi^{2}(7)=8352, p<\right.$ $.001)$ as well as the Risky-Risky problems $\left(\chi^{2}(7)=733.2, p<.001\right)$.

Panel C of Figure 1, shows the sample size distributions based on the type of outcomes involved in the problem: gains, losses, and mixed (gains and losses). Again humans and model agents are very similar in gain problems (Human: median $=20$, mean $=23.51, \mathrm{SD}=16.45 ; \mathrm{IBL}$ 
model: median $=19$, mean $=21.35, \mathrm{SD}=14.11)$; in loss problems $($ Human: median $=18$, mean $=21.25, \mathrm{SD}=15.34 ; \mathrm{IBL}$ model: median $=20$, mean $=21.81, \mathrm{SD}=13.55)$; and in the mixed problems $($ Human: median $=19$, mean $=22.28, \mathrm{SD}=15.14 ; \mathrm{IBL}$ model: median $=18$, mean $=$ 20.44, $\mathrm{SD}=13.58$ ). The chi-square test signifies that human sample distribution and model sample distribution is strongly associated in all the domains (Gain: $\chi^{2}(7)=2311.1, \mathrm{df}=7, \mathrm{p}<$ 0.001, Loss: $\chi^{2}(7)=1611.8, \mathrm{df}=7, \mathrm{p}<0.001$, and Mixed: $\left.\chi^{2}(7)=478, \mathrm{df}=7, \mathrm{p}<0.001\right)$. 


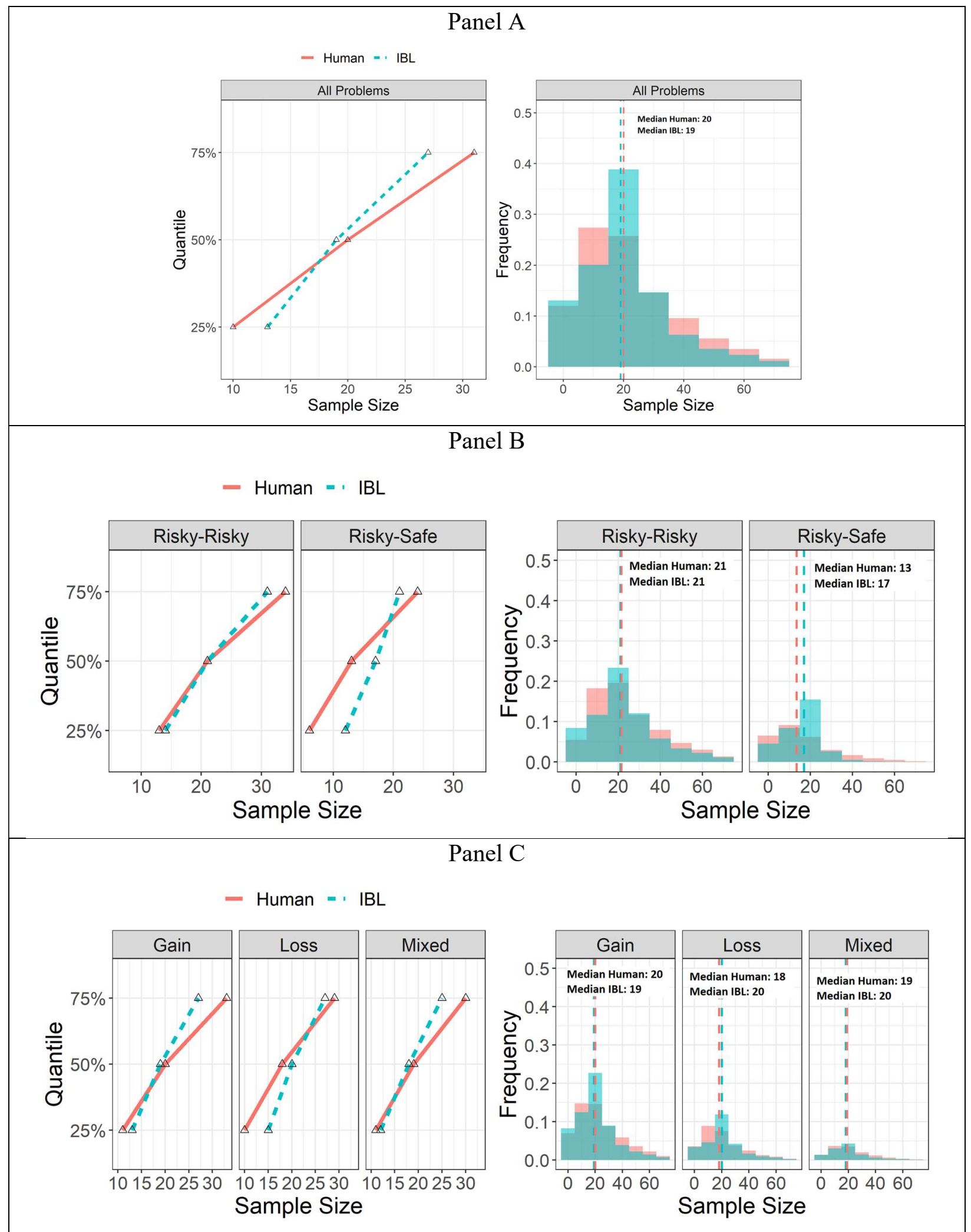

Figure 1. The distribution of sample sizes from the human data (red) and IBL model (blue) for a) all problems, b) Risky-Risky and Risky-Safe problems, and c) Gains, Losses and Mixed domain problems. 


\section{Analysis of Sample Size per Paper-Problem Pair.}

To investigate the model predictions and the human data in more detail, we analyzed the relationships between the sample size in human data and in the IBL agents for each of the 1,041 paper-problem pairs.

Figure 2 shows these correlations for all problems (Panel A), for Risky-Risky and RiskySafe problems (Panel B), and for Gains, Losses, and Mixed problems (Panel C). The overall sample size per paper-problem pair predicted by the IBL model is positively correlated to the sample size observed in the human data $(\mathrm{R}=0.26)$. This is a very high correlation considering that the IBL model was not fit in any way to the human data and considering the large variety of problems in the human data. As shown by the diagonal red line, the IBL model under-samples compared to humans (i.e., the majority of the observations are below the red line). The IBL model is positively correlated to the sample size observed in the human data for Risky-Risky problems $(R=0.023)$ and negatively correlated for Risky-Safe problems $(R=-0.21)$; and positively correlated to all three domains including gains, losses and mixed. In general, humans sampled significantly more in the Risky-Safe type of problems than what the IBL model predicts. 


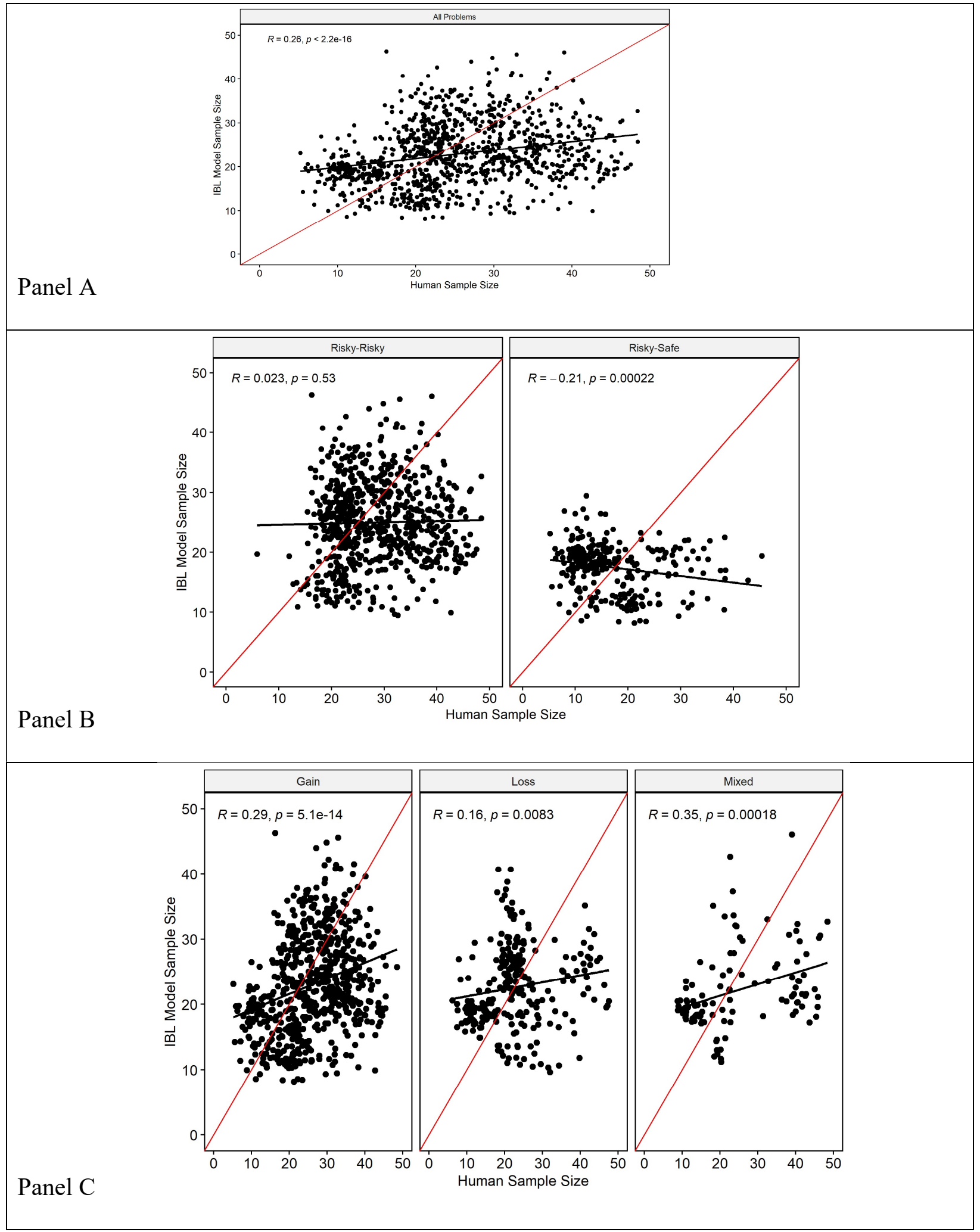

Figure 2. Correlation for each of the 1,041 paper-problem pairs as predicted by the IBL model and the observed sample size in the human data. Panel A shows all the problems; Panel B shows the risky and safe problems, and Panel $\mathrm{C}$ shows the Gains, Loss and Mixed problems. 


\section{Delta Gap Over Sample Count.}

Here we illustrate the $\delta G a p_{t}$ over the sampling process. Again, the expectation is that the $\delta G a p_{t}$ will decrease over samples. The intuition is that as more samples are accumulated, the predicted outcomes of each of the two options (by Blended values in Equation 3) will be close to the actual outcome observed in each option. That is, the predicted error, $\delta G a p_{t}$, will decrease over time. However, the $\delta G a p_{t}$ might not always decrease monotonically, as it would depend on the statistical structure of the gambles.

To have a frame of gross reference for the $\delta$ Gap $_{t}$ in the human data, we calculated an experienced value of each of the two options ExV using the frequencies of each outcome the humans observed. In past research, this has been shown to be an approximation to the Blended value of the IBL model in choice tasks (Gonzalez \& Mehlhorn, 2016). ExV for each option $k$ is defined as follows:

$$
\operatorname{ExV}_{k}=\sum_{i=1}^{n} \frac{\text { tot } \quad \text { occurance of outcome } \text { in option } k}{\text { total occurance of option } k} * \text { Outcome }_{i k}
$$

Using the ExV, we calculate the Gap (Equation 4) and marginal value $\delta$ Gap $_{t}$ (Equation 5) using human data.

Given the large differences across problems in terms of the outcome values, to average the $\delta G a p_{t}$ over the sample count, we normalized the values by the following function:

$$
\operatorname{Norm}\left(\delta G a p_{t}\right)=\frac{\delta G a p-\min (\delta G a p)}{\max (\delta G a p)-\min (\delta G a p)}
$$

Figure 3 illustrates the normalized $\delta G a p_{t}$ in human and the model data, plotted up to the overall sample size median of 21 (data becomes noisier with more samples, as the number of observations decrease). The $\delta G a p_{t}$ clearly decreases over samples, and the human data's proxy 
for the $\delta \mathrm{Gap}_{t}$ shows that decrease too. For the model data, the $\delta G a p_{t}$ starts very large and decreases rapidly in the first samples. The initial large values of $\delta G_{a p}$ in the model are due to our assumptions of pre-populated values in the instances: Basically, for the IBL model, every experienced outcome will be maximally different from expectations in the initial trials, given general assumptions made in the model about initial expectation values (Lejarraga et al., 2012). 


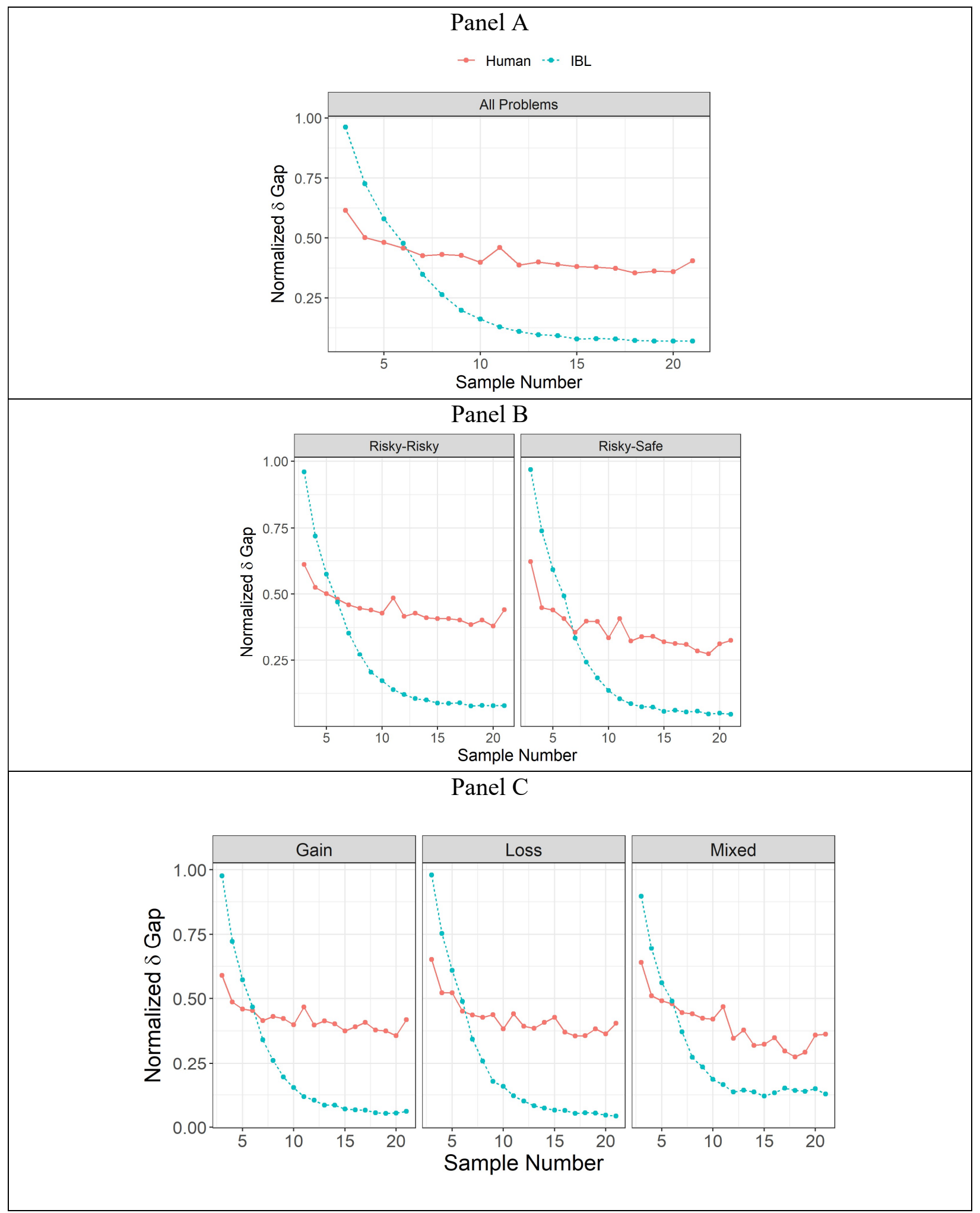

Figure 3: An example of the marginal value $\delta G a p_{t}$ in human and model data up to 21 samples. 


\section{Proportion of Maximization During Sampling and Choice.}

To illustrate that in addition to capture the sample size, the IBL model is capable of capturing the decisions during sampling and the consequential choice, we calculated the proportion of maximization (PMax) during sampling and final choice. The PMax represents the number of times the option with the maximum objective expected value was selected. Table 1 presents the PMax during sampling and during the final choice in the human dataset and the IBL model data set for all the problems, and using the same groupings as in the sections above. The RMSE are all very low (ranging from 0.02 to 0.06 ), suggesting that the model is generally able to capture the PMax during sampling and choice. This result replicates the well-known capability of IBL models to capture choices during sampling and after sampling (e.g., Gonzalez \& Dutt, 2011; Lejarraga et al., 2012).

Table 1: PMax in Sampling and Final Choice phases for human and model data.

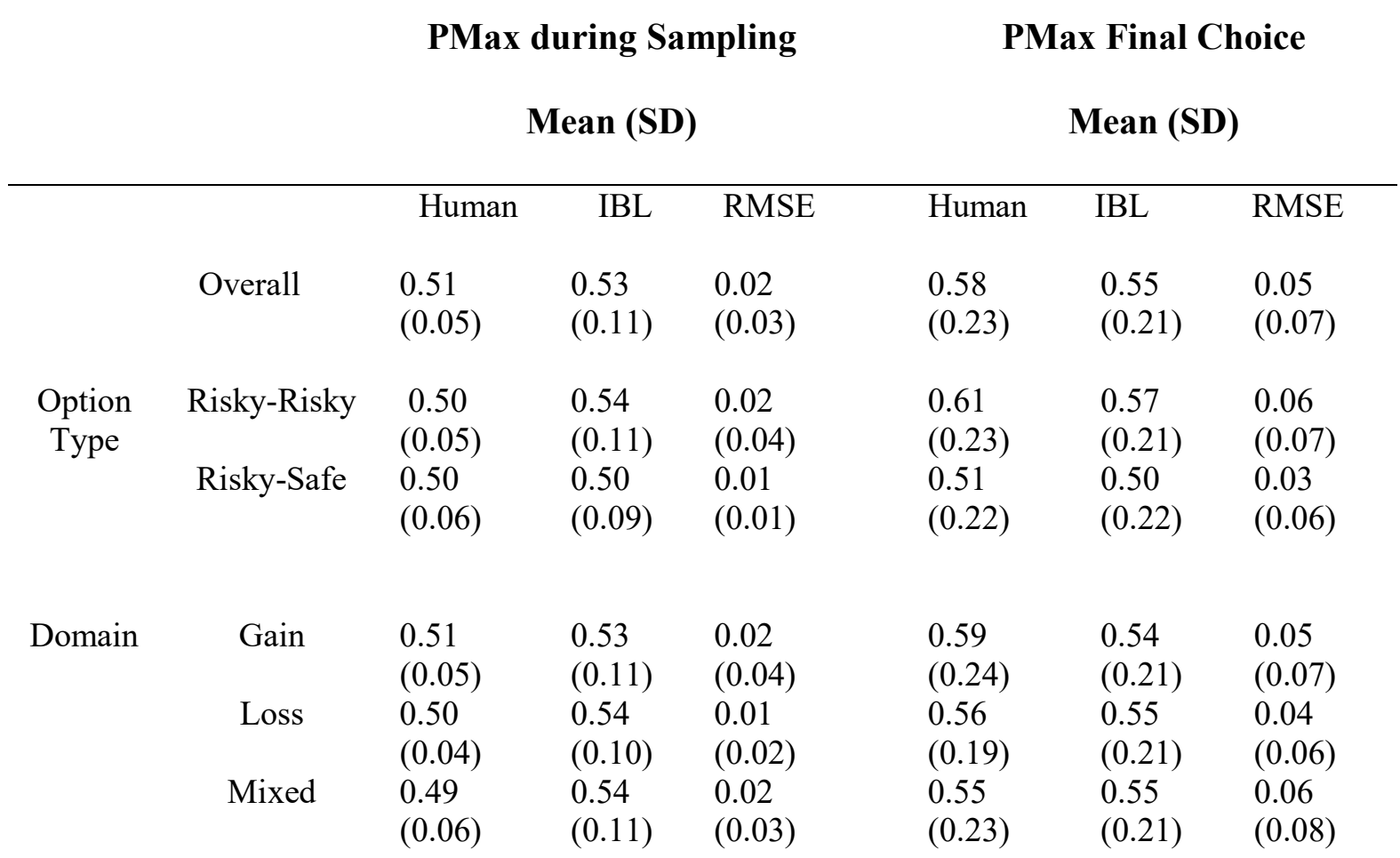




\section{Discussion}

Understanding how people decide the length of their exploration process (i.e., how to make a stopping decision) before making a choice, is critical for theories of decisions from experience. The sampling paradigm in DfE is a simple but useful way to study this important question. Research on the sampling paradigm has addressed related questions, for example: how the choice is influenced by the information collected during sampling, and what are the moderators for the sampling phase that influence the decision of the number of samples to take. These moderators include: the variability of the problems (e.g., whether the choice is being made between two risky alternatives or a risky and a safe alternative) (Wulff et al., 2018; Gonzalez \& Mehlhorn, 2016); the motivations and costs during sampling (Hau et al 2008); the loss or gain domain of the problems (Lejarraga et al., 2012; Gonzalez \& Mehlhorn, 2016); and others.

But the question of how people decide when to stop sampling, and what are the cognitive mechanisms involved in such decision, has received very little attention. Theories that are able to integrate the whole process of decisions from experience, from the sequential decisions from sampling, the decision of when stop sampling, and the subsequent choice into an integrated model do not exist. The IBL model of binary choice is the only current available option able to achieve such integrated theoretical account of DfE. IBL has already been considered an important integrated cognitive account of DfE (Hertwig, 2015; Wulff et al., 2018), as it has demonstrated generalized predictions on various DfE paradigms, accounting for individual choices during sampling and final choice (Gonzalez \& Dutt, 2011; Lejarraga et al., 2012; Gonzalez \& Mehlhorn, 2016; Dutt \& Gonzalez, 2015). The current paper advances this model by 
proposing a satisficing mechanism to account for the decision of when to stop sampling, while using the same IBL choice mechanisms.

The satisficing process proposed here differs from other attempts to model stopping decisions in the sampling paradigm (Markant et al., 2015; Srivastava et al., 2016). Mainly, our proposed prediction error mechanism for stopping decisions, $\delta G a p_{t}$, relies directly on Blending, the belief value of each of the options that is already part of IBLT (Gonzalez et al., 2003). This occurs by tracking the differences of the prediction error between options over the samples. This concept is similar to that of "volatility" discussed in Srivastava et al., (2016), but instead of using completely new formulations of the concept of prediction error, we use the same Blending mechanism in IBLT that is used to make a choice.

Importantly, our model is able to capture stopping decisions of individuals in the largest data set known of the sampling paradigm (Wulff et al 2018). In contrast to past research (Markant et al., 2015; and Srivastava et al., 2016), we did not fit the model to human data. We also did not make any unreasonable assumptions regarding information that participants did not have in the studies. That is, our model's results are pure predictions from the theoretical assumptions of IBL, a cognitive plausible model of DfE. Considering this, our results are an excellent account of the human decisions. The IBL model is able to capture the sample size distributions from humans and to predict the sampling sequence lengths for individuals with significantly more accuracy than past models. Our model's prediction of individual sampling size is $\mathrm{R}=0.26$ (compared to $\mathrm{R}=0.03$ in Srivastava et al., 2016). In addition, our model is able to capture individual choice per sample, and choice from experience, after sampling. Thus, our model provides an integrated theoretical account of DfE in the sampling process. The general integration of sampling processes with stopping decisions and choice has already been discussed 
as part of IBLT (Gonzalez et al., 2003). However, in this general theory, stopping decisions are determined by externally set deadlines and time constraints. In the sampling paradigm people are not provided with deadlines, sequence length information, or time constraints to make a stopping decision. Thus, we propose an endogenously-generated stopping mechanism that applies in each sample taken in the task; the mechanism is based on the relative prediction error of the alternatives using the IBLT's choice rule, Blending.

The work presented in this chapter is only the beginning of what we expect to be an interesting integration with other models of sequential learning and decisions from sampling. First, some of our current assumptions will need to be investigated further. For example, in this work we assumed a "close to zero" threshold to compare to the $\delta G a p_{t}$. But, it is very possible that this threshold is problem-dependent and individual-dependent. We also assumed a probabilistic non-linear increasing function for stopping every time the threshold is reached. These two assumptions will require more investigation to determine an individualized generation of such thresholds according to the individual experiences. Generally, the results presented here did not rely on human data; and in particular the specific sequences observed by the human participants. We suspect that feeding the IBL model with the same sequence of experiences from each individual would make our model significantly more accurate in the prediction of the sampling size. These and other questions are left for future research.

Second, in many models of sequential decision making, the concept of a "Threshold" is used to determine the decision of when to stop sequential choice (Baunmann et al., 2020; Guan et al., 2020). Often, in this research the question is about the deviations of human thresholds and optimal thresholds. These models often rely on pre-determined sequences from defined distributions and specific lengths. In these experiments, participants commonly know the length 
of the sequence (Baunmann et al., 2021; Guan et al., 2020). This information significantly changes the nature of the problem compared to the sampling paradigm, where participants are free to sample for as long as they want without any total sample limit or information. Thus, the generality of our proposed mechanism to such sequential choice tasks will need to be investigated.

\section{Authors' Note:}

This research was supported by the Air Force Research Laboratories Award FA8650-20F-6212 sub-award number 1990692 and by the Army Research Office, Network Science Program, Award Number: W911NF1710431. We thank Jeffrey Flagg for editorial review. Correspondence concerning this article should be addressed to Cleotilde Gonzalez, Dynamic Decision Making Laboratory, Carnegie Mellon University, Pittsburgh, PA 15213. E-mail: coty@cmu.edu.

Data, scripts for analyses, and the IBL model's code are available in Open Science Framework: https://osf.io/b3mfk/?view_only=847aa9221d994793bb450b50d271c883.

\section{References}

Anderson, J. R., Bothell, D., Byrne, M. D., Douglass, S., Lebiere, C., \& Qin, Y. (2004). An integrated theory of the mind. Psychological review, 111(4), 1036.

Baumann, C., Singmann, H., Gershman, S. J., \& von Helversen, B. (2020). A linear threshold model for optimal stopping behavior. Proceedings of the National Academy of Sciences, 117(23), 12750-12755. https://doi.org/10.1073/pnas.2002312117

Busemeyer, J. R., \& Townsend, J. T. (1993). Decision Field Theory: A dynamic cognitive approach to decision making. Psychological Review, 100, 432-459.

https://doi.org/10.1037/0033-295x.100.3.432 
Dutt, V., \& Gonzalez, C. (2015). Accounting for outcome and process measures in dynamic decision-making tasks through model calibration. Carnegie Mellon University Pittsburgh United States. https://doi.org/10.11588/jddm.2015.1.17663

Erev, I., Ert, E., Roth, A. E., Haruvy, E., Herzog, S., Hau, R., Hertwig, R., Stewart, T., West, R., \& Lebiere, C. (2010). A choice prediction competition for choices from experience and from description. Journal of Behavioral Decision Making, 23, 15-47. https://doi:10.1002/bdm.683

Gonzalez, C. (2013). The boundaries of instance-based learning theory for explaining decisions from experience. In V. S. Chandrasekhar Pammi \& N. Srinivasan (Eds.), Progress in brain research (Vol. 202, pp. 73-98). Amsterdam, Netherlands: Elsevier. https://doi.org/10.1016/b978-0-444-62604-2.00005-8

Gonzalez, C., Ben-Asher, N., Martin, J. M., \& Dutt, V. (2015). A cognitive model of dynamic cooperation with varied interdependency information. Cognitive science, 39(3), 457-495. https://doi.org/10.1111/cogs.12170

Gonzalez, C., \& Dutt, V. (2011). Instance-Based Learning: Integrating sampling and repeated decisions from experience. Psychological Review, 118(4), 523-551. https://doi: $10.1037 / \mathrm{a} 0024558$

Gonzalez, C., \& Dutt, V. (2016). Exploration and exploitation during information search and consequential choice Journal of Dynamic Decision Making, 2(2),1-8.

Gonzalez, C., Lerch, J. F., \& Lebiere, C. (2003). Instance-based learning in dynamic decision making. Cognitive Science, 27(4), 591-635. https://doi: 10.1016/S0364-0213(03)00031-4 
Gonzalez, C., \& Mehlhorn, K. (2016). Framing from experience: Cognitive processes and predictions of risky choice. Cognitive science, 40(5), 1163-1191. https://doi.org/10.1111/cogs.12268

Guan, M., Stokes, R., Vandekerckhove, J., \& Lee, M. D. (2020). A cognitive modeling analysis of risk in sequential choice tasks. Judgment and Decision Making, 15(5), 823-850. https://doi.org/10.31234/osf.io/evzp9

Hau, R., Pleskac, T. J., Kiefer, J., \& Hertwig, R. (2008). The description-experience gap in risky choice: The role of sample size and experienced probabilities. Journal of Behavioral Decision Making, 21(5), 493-518. https://doi.org/10.1002/bdm.598

Hertwig, R. (2015). Decisions from experience. The Wiley Blackwell handbook of judgment and decision making, 2, 239-267. https://doi.org/10.1002/9781118468333.ch8

Hertwig, R., Barron, G., Weber, E. U., \& Erev, I. (2004). Decisions from experience and the effect of rare events in risky choice. Psychological Science, 15, 534-539. https://doi: 10.1111/j.0956-7976.2004.00715.x

Hertwig, R., \& Erev, I. (2009). The description-experience gap in risky choice. Trends in Cognitive Sciences, 13(12), 517-523. https://doi: 10.1016/j.tics.2009.09.004

Hertwig, R., \& Pleskac, T. J. (2010). Decisions from experience: Why small samples? Cognition, 115, 225-237. https://doi.org/10.1016/j.cognition.2009.12.009

Lebiere, C. (1999). A blending process for aggregate retrievals. In Proceedings of the $6^{\text {th }}$ ACT-R Workshop. George Mason University, Fairfax, VA.

Lejarraga, T., Dutt, V., \& Gonzalez, C. (2012). Instance-based learning: A general model of repeated binary choice. Journal of Behavioral Decision Making, 25(2), 143-153. https://doi.org/10.1037/e722992011-088 
Lejarraga, T., \& Gonzalez, C. (2011). Effects of feedback and complexity on repeated decisions from description. Organizational Behavior and Human Decision Processes, 116(2), 286295. https://doi.org/10.1016/j.obhdp.2011.05.001

Lejarraga, T., Hertwig, R., \& Gonzalez, C. (2012). How choice ecology influences search in decisions from experience. Cognition, 124(3), 334-342. https://doi.org/10.1016/j.cognition.2012.06.002

Lejarraga, T., Lejarraga, J., \& Gonzalez, C. (2014). Decisions from experience: How groups and individuals adapt to change. Memory \& cognition, 42(8), 1384-1397. https://doi.org/10.3758/s13421-014-0445-7

March, J. G., \& Simon, H. A. (1958). Organizations. Wiley, New York.

Markant, D., Pleskac, T. J., Diederich, A., Pachur, T., \& Hertwig, R. (2015). Modeling choice and search in decisions from experience: A sequential sampling approach. In 37 th annual meeting of the cognitive science society (pp. 1512-1517). Cognitive Science Society.

Simon, H. (1957). A behavioral model of rational choice. Models of man, social and rational: Mathematical essays on rational human behavior in a social setting, 241-260.

Srivastava, N., Müller-Trede, J., Schrater, P. R., \& Vul, E. (2016). Modeling sampling duration in decisions from experience. In Proceedings of the 38th Annual Meeting of the Cognitive Science Society.

Tversky, A., \& Kahneman, D. (1992). Advances in prospect theory: Cumulative representation of uncertainty. Journal of Risk and uncertainty, 5(4), 297-323.

Wulff, D. U., Mergenthaler-Canseco, M., \& Hertwig, R. (2018). A meta-analytic review of two modes of learning and the description-experience gap. Psychological bulletin, 144(2), 140. https://doi.org/10.1037/bul0000115 